\title{
Polyelectrolyte multilayered nanoparticles: using nanolayers for controlled and targeted systemic
}

\section{release}

"The concept of generating drug delivery nanoparticles that provide controlled, staged and/or triggered release of different drugs at appropriate points in the body is highly attractive..."

\section{KEYWORDS: blood stability $\approx$ cancer $\approx$ layer-by-layer $\approx$ molecular targeting $\approx$ multilayer - passive targeting " polyelectrolyte $\approx$ polymer nanoparticle $\approx$ systemic delivery - targeted nanoparticle}

The simplicity of layer-by-layer (LbL) assembly [1] - a method that involves the alternating adsorption from water of two or more polymers or other multivalent species with complementary interactions - has led to a recent and greatly accelerated growth of new drug delivery systems that take advantage of the mild aqueous conditions and high drug loadings that are possible using this method [2-4]. The ability to incorporate a broad range of therapeutic molecules at meaningful drug loadings, from small molecule drugs to sensitive biologics such as peptides, proteins, growth factors and nucleic acids has contributed significantly to this revolution. Furthermore, there is significant promise in the ability to release multiple drugs from a singular nanoscale thin film with independent release profiles of each drug tuned to the desired pharmacokinetics [5]. This promise is furthered by recent demonstrations that multilayer thin films can regulate not only relative release behavior, but yield time-sequenced release from surfaces [6,7]. Many exciting new applications involve the use of coated micron-sized particles and microcapsules that are of interest for localized release biomaterials, cellular mimics and the targeting of cells that readily engage with micronscale particles, such as macrophages and certain immunological cells [8].

The advantages described above are highly relevant to the goals of targeted nanomedicine, in which the nanoscale nature of the delivery vehicle itself enables accumulation of drug carriers in pathological tissues and access to tissues via various modes of cell penetration and entry. The concept of generating drug delivery nanoparticles that provide controlled, staged and/or triggered release of different drugs at appropriate points in the body is highly attractive to the treatment of cancer, infectious disease, lung, cardiovascular and neurological conditions, and numerous other applications. One example of particular interest to the research in our laboratories is the release of agents that lower the tumor's drug resistance, followed synergistically by the release of potent chemotherapy agents. Despite the many promising accomplishments achieved in LbL delivery, the ability to generate LbL-based nanoparticles that can be delivered directly through the bloodstream has remained an important and critical challenge. As outlined below, new breakthroughs in the past few years allow us to better manipulate the LbL of nanoparticles in the size range appropriate for nanomedicine, and to address the important issues of stability, stealth characteristics and biodistribution that are key to systemic delivery. As discussed here, bringing the many advantages of LbL down to the range of sub-100-nm scale carriers opens up many possibilities toward new and critical applications in the field of nanomedicine.

The ability to controllably adsorb polyelectrolyte multilayers onto sub-100-nm nanoparticles was effectively demonstrated with concentrated suspensions of gold colloids as small as $13 \mathrm{~nm}$ with alternating layers of a polycation (polyallylamine hydrochloride) and polyanion (sulfonated polystyrene); 20 layers of LbL film produced a uniform shell thickness of $7.5 \mathrm{~nm}$ [9]. By adsorbing a final layer of poly(hydroxypropylmethacrylamide), it is possible to impart a hydrated stealth layer to the nanoparticle that greatly lowered association and uptake in macrophages in cell culture [10]. The incorporation of siRNA cargo has also been recently demonstrated on the surfaces of gold nanoparticles using electrostatic LbL assembly [1] with polyethyleneimine (PEI), well known for its ability to transfect cells in polyplexes formed with siRNA and DNA. This study only examines a single bi- or a tri-layer of siRNA and PEI; however,

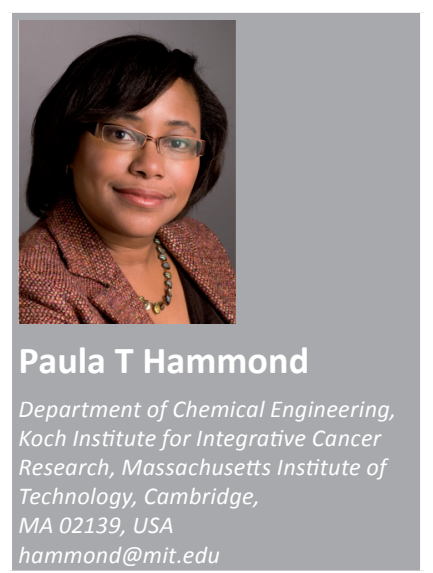

$\begin{array}{ll}\text { Future } & \text { fSO }\end{array}$ 
it illustrates that a single layer of adsorbed siRNA introduces close to 800 siRNA molecules per $16-\mathrm{nm}$ gold nanoparticle, which is equivalent in loading to a covalently attached siRNA gold nanoparticle. The siRNA was released in an active form in vitro when incubated with $\mathrm{CHO}-\mathrm{K} 1$ cells; on the other hand, the low efficacy of its release suggested a need to devise multilayer systems that fully disassemble and release the active component once the cargo has entered the cell. Another study investigated the direct encapsulation of DNA-PEI polyplexes with LbL films and reported enhanced in vitro luciferase gene transfection [12].

"...bringing the many advantages of layer-bylayer down to the range of sub-100-nm scale carriers opens up many possibilities toward new and critical applications in the field of nanomedicine."

These important and fairly recent developments provide an indication of the potential for LbL nanoparticles in nanomedicine, but do not demonstrate systemic delivery in vivo. The stability of an electrostatically assembled nanoparticle in the presence of high concentrations of serum proteins, a physiological abundance of proteases and the mechanical shear forces of the blood stream during biodistribution are key barriers to the use of LbL nanodelivery systems. We set out to systematically investigate $\mathrm{LbL}$ nanoparticles that can withstand the much more aggressive environment of the bloodstream, and design systems that can significantly enhance the biodistribution of a nanoparticle while passively targeting the tumor [13]. These first studies for systemic in vivo delivery of LbL nanoparticles were performed using real-time live animal imaging of the nanoparticles, tracking the accumulation and clearance of 20-nm quantum dots layered with a fluorescently tagged poly-L-lysine (PLL) in alternation with a range of negatively charged biologically derived polysaccharides. We found that the number of LbL bilayers adsorbed onto a nanoparticle greatly influences its stability in the body [13]. The adsorption of just a single bilayer leads to rapid disassembly and clearance of nanoparticles within $30 \mathrm{~min}$. On the other hand, when a minimum number of six bilayers are adsorbed, we observed significant and meaningful improvements in clearance rates of the nanoparticles up to several hours; furthermore, the quantum dot and PLL were tracked together, indicating that the nanoparticle LbL assembly remains intact during circulation.

There was also a large impact of polyion used for the final deposited outer layer [13]. As expected, nanoparticles topped with a polycation exhibited rapid macrophage uptake and clearance through the reticuloendothelial system through nonspecific interaction of the positive charge with the negatively charged cell membrane, and the adsorption of opsonins and serum proteins. By contrast, LbL nanoparticles topped with a polyanion indicated extended blood circulation halflives that were significantly higher in all cases. The negatively charged nanoparticles also illustrated very effective and sustained accumulation in mouse tumors due to the enhanced permeation and retention (EPR) effect, with long residence times of approximately $18 \mathrm{~h}$ within the tumor. Surprisingly meaningful differences were found between the blood circulation half-life of some of the common polysaccharides such as dextran sulfate in comparison with the native extracellular component, hyaluronic acid. Liver uptake as low as $10-15 \%$ was observed in the hyaluronic acid outer layer nanoparticles in mice with and without tumors, outperforming the high degree of liver accumulation observed in many previously reported traditional drug delivery carriers. We now believe these differences may be attributed to specific binding interactions within the liver, or the degree of hydration and bound water present in the polyelectrolyte brush formed as the top layer. Because accumulation in the liver is one of the key issues that face the drug delivery nanoparticle field, further understanding of the role of polyelectrolytes as stealth or shield layers should lead to promising therapeutic applications and a more rapid translation to clinical application. Polyelectrolyte assembly can be used as a versatile means to enable triggered response in the tumor microenvironment; we took advantage of the fact that positive charge mediates nonspecific but highly effective cell uptake by constructing an LbL system in which the outermost layer is a hydrated stealth polymer layer bound to an underlying positively charged film [14]. Most solid tumors are slightly to highly hypoxic; this hypoxia leads to a localized decrease in $\mathrm{pH}$ that can be exploited for delivery applications. In this case, a $\mathrm{pH}$-responsive iminobiotin molecule was used to attach PLL to a monofunctional polyethylene glycol stealth layer via iminobiotin-avidin linkages. The polyethylene glycol provides a hydrated cloak layer for the nanoparticle as it travels through the blood stream without undesired and nonspecific uptake by macrophages or nontargeted cells. Once the nanoparticle reaches the tumor, the iminobiotin-avidin linkage breaks apart due to the slightly lower $\mathrm{pH}$, and the stealth cloak layer is 'shed', leaving behind the positively charged PLL layer to 
mediate tumor cell entry. In animal experiments, these nanoparticles accumulated preferentially in subcutaneous tumors due to enhanced cellular uptake, and at greater than $48 \mathrm{~h}$, well past the time period when the EPR effect is a factor, significant retention was observed, indicating that preferential uptake of these nanoparticles can be mediated through the design of LbL nanoparticles to be responsive to specific environments. We now hope to move this simple development toward responsive behavior in other targeted tissues, triggered by $\mathrm{pH}$, reduction/oxidation, and temperature, for engaging cells and inducing uptake and delivery.

\section{"Generation of newer and simpler methods that enable rapid manufacture are greatly needed; along with these new methods comes the need for high reproducibility and uniformity."}

Since our reports on the in vivo behavior of LbL nanoparticles, additional work has appeared in this area. These include the design of a drugloaded mesoporous silica nanoparticles coated with a cisplatin-containing LbL film with a $\mathrm{pH}$ responsive polymer that unloads both drug molecules at acidic conditions [15], and the in vitro characterization of LbL nanoparticles with a core loaded with paclitaxel and a surface functionalized with specific molecular targeting ligands [16]. A mouse tumor model study on LbL-coated pluronic micelles loaded with paclitaxel has recently demonstrated improvements in suppression of tumor growth compared to free drug and the micelle alone [17], and additional siRNA systems are being developed [18], indicating the ability of the LbL approach to stabilize nanoparticle systems, control drug release and confirming their ability to optimize nanoparticle biodistribution. We have recently demonstrated that even a single polyelectrolyte layer can be a powerful tool in the condensation of highly organized siRNA structures; by wrapping a high-molecular-weight siRNA macromolecule generated using rolling circle transcription, we can form a self-assembled micron-scale siRNA vehicle that is readily shrunk to nanometer length scales with the adsorption of a polycation [19]. There are clear implications in the use of LbL approaches to further modify such amplified siRNA or DNA structures to enhance biodistribution and delivery.

Some challenges remain to establish the use of LbL nanoparticles for biomedical applications. Although the current means of colloidal adsorption followed by centrifugation and wash steps has proven effective, it is also time-consuming, prone to low yields, and less adaptable to common manufacturing processes. There are already signs of new innovative means to generate polyelectrolyte-coated nanomaterials. Simultaneous sonication-assisted breakup of hydrophobic drug nanocrystals with polyelectrolyte adsorption yields coated nanocrystals in a process that eliminates rinse and centrifugation steps [20], and a new surface acoustic wave method uses the atomization of polyelectrolytes from solution to generate alternating polyelectrolyte complexes in-flight [21]. Generation of newer and simpler methods that enable rapid manufacture are greatly needed; along with these new methods comes the need for high reproducibility and uniformity. The in vivo compatibility of LbL nanoparticle systems has been reported to be quite high; however, as these new nanomaterials are generated in smaller and more diverse forms, extensive toxicological examinations will also prove necessary.

On the other hand, there are many opportunities in this new and quickly growing area. A more in-depth understanding of the ways in which LbL nanoparticle systems may be designed for triggered or passive, sequential release will yield systems that greatly surpass those of traditional nanoparticles. Future LbL nanoparticle systems may take advantage of the high $\mathrm{pH}$ sensitivity of certain hydrogen bond donor-acceptor groups or reversible biological bonds such as disulfides. Cell receptor-mediated interactions for molecular targeting are highly attainable for LbL systems and may lead to novel approaches to cell-stimulated release mechanisms. The field of LbL nanoparticle assembly is at its nascence, and the ability to show the potential of these unique nanolayered materials and their behavior in vivo firmly establishes their potential for nanomedicine. With the continued development of these systems, and the careful examination of their properties - both in vitro and in vivo - it is expected that the next few years will significantly shape the direction and opportunities for translational medicine, enabling realization of the potential that these systems provide.

Financial \& competing interests disclosure PT Hammond is funded by NIH NIBIB grant R01EB008082. The author has no other relevant affiliations or financial involvement with any organization or entity with a financial interest in or financial conflict with the subject matter or materials discussed in the manuscript apart from those disclosed.

No writing assistance was utilized in the production of this manuscript. 


\section{References}

1 Decher G. Fuzzy nanoassemblies: toward layered polymeric multicomposites. Science 277, 1232 (1997).

2 Hammond PT. Engineering materials layer-by-layer: challenges and opportunities in multilayer assembly. AIChE J. 57(11), 2928-2940 (2011).

3 Pavlukhina S, Sukhishvili S. Polymer assemblies for controlled delivery of bioactive molecules from surfaces. Adv. Drug Deliv. Rev. 63(9), 822-836 (2011).

4 Boudou T, Crouzier T, Ren KF, Blin G, Picart C. Multiple functionalities of polyelectrolyte multilayer films: new biomedical applications. Adv. Mater. 22(4), 441-467 (2010).

5 Wood KC, Chuang HF, Batten RD, Lynn DM, Hammond PT. Controlling interlayer diffusion to achieve sustained, multiagent delivery from layer-by-layer thin films. Proc. Natl Acad. Sci. USA 103(27), 10207-10212 (2006).

6 Kim BS, Smith RC, Poon Z, Hammond PT. $\mathrm{MAD}$ (multiagent delivery) nanolayer: delivering multiple therapeutics from hierarchically assembled surface coatings. Langmuir 25, 14086-14092 (2009).

7 Hong J, Shah NJ, Drake AC et al. Graphene multilayers as gates for multi-week sequential release of proteins from surfaces. ACS Nano 6(1), 81-88 (2012).

8 Becker AL, Johnston APR, Caruso F. Layer-by-layer-assembled capsules and films for therapeutic delivery. Small 6(17), 1836-1852 (2010).

9 Schneider G, Decher G. From functional core/shell nanoparticles prepared via layer-by-layer deposition to empty nanospheres. Nano Lett. 4(10), 1833-1839 (2004).

10 Schneider GF, Subr V, Ulbrich K, Decher G. Multifunctional cytotoxic stealth nanoparticles. a model approach with potential for cancer therapy. Nano Lett. 9(2), 636-642 (2009).

11 Elbakry A, Zaky A, Liebkl R, Rachel R, Goepferich A, Breunig M. Layer-by-layer assembled gold nanoparticles for siRNA delivery. Nano Lett. 9(5), 2059-2064 (2009).

12 Saul JM, Wang CHK, Ng CP, Pun SH. Multilayer nanocomplexes of polymer and DNA exhibit enhanced gene delivery. $A d v$. Mater. 20(1), 19-25 (2008).

13 Poon Z, Lee JB, Morton SW, Hammond PT. Controlling in vivo stability and biodistribution in electrostatically assembled nanoparticles for systemic delivery. Nano Lett. 11(5), 2096-2103 (2011).

14 Poon Z, Chang D, Zhao XY, Hammond PT. Layer-by-layer nanoparticles with a $\mathrm{pH}$ sheddable layer for in vivo targeting of tumor hypoxia. ACS Nano 5(6), 4284-4292 (2011).

15 Wan XJ, Zhang GY, Liu SY. pH-Disintegrable polyelectrolyte multilayer-coated mesoporous silica nanoparticles exhibiting triggered co-release of cisplatin and model drug molecules. Macromol. Rapid Commun. 32(14), 1082-1089 (2011).

16 Yu X, Pishko MV. Nanoparticle-based biocompatible and targeted drug delivery: characterization and in vitro studies. Biomacromolecules 12(9), 3205-3212 (2011).

17 Yuk SH, Oh KS, Cho SH et al. Enhancement of the targeting capabilities of the paclitaxelloaded pluronic nanoparticles with a glycol chitosan/heparin composite. Mol. Pharm. 9(2), 230-236 (2012).

18 Lee M-Y, Park S-J, Park K, Kim K-S, Lee H, Hahn SK. Target-specific gene silencing of layer-by-layer assembled gold cysteamine/ siRNA/PEI/HA nanocomplex. ACS Nano 5(8), 6138-6147 (2011).

19 Lee JB, Hong J, Bonner DK, Poon Z, Hammond PT. Self-assembled RNA interference microsponges for efficient siRNA delivery. Nat. Mater. 11(4), 316-322 (2012) (Epub ahead of print).

20 Pattekari P, Zheng Z, Zhang X, Levchenko T, Torchilin V, Lvov Y. Top-down and bottom-up approaches in production of aqueous nanocolloids of low solubility drug paclitaxel. Phys. Chem. Chem. Phys. 13(19), 9014-9019 (2011).

21 Qi AS, Chan P, Ho J, Rajapaksa A, Friend J, Yeo LL. Template-free synthesis and Encapsulation technique for layer-by-layer polymer nanocarrier fabrication. ACS Nano 5(12), 9583-9591 (2011). 